\title{
T stage-dependent lymph node and distant metastasis and the accuracy of lymph node assessment in rectal cancer
}

\author{
Henry Ptok · Frank Meyer · Roland S. Croner · Ingo Gastinger · Benjamin Garlipp
}

Received: 23 November 2020 / Accepted: 9 April 2021 / Published online: 12 May 2021

(C) The Author(s) 2021

\begin{abstract}
Summary
Objective To analyze data obtained in a representative number of patients with primary rectal cancer with respect to lymph node diagnostics and related tumor stages.

Methods In pT2-, pT3-, and pT4 rectal cancer lesions, the impact of investigated lymph nodes on the frequency of $\mathrm{pN}+$ status, the cumulative risk of metachronous distant metastases, and overall survival was studied by means of a prospective multicenter observational study over a defined period of time.

Results From 2000 to 2011, the proportion of surgical specimens with $\geq 12$ investigated lymph nodes increased significantly, from $73.6 \%$ to $93.2 \%(p<0.001$; the number of investigated lymph nodes from 16.2 to 20.8; $p<0.001)$. Despite this, the percentage of $\mathrm{pN}+$ rectal cancer lesions varied only non-significantly (39.9\% to $45.9 \%$; $p=0.130$; median, $44.1 \%$ ). For pT2-, pT3-, and pT4 rectal cancer lesions, there was an increasing proportion of $\mathrm{pN}+$ findings correlating significantly with the number of investigated lymph nodes up to $n=12$ investigated lymph nodes. Only in pT3 rectal cancer was there a significant increase in $\mathrm{pN}+$ findings in case of $>12$ lymph nodes $(p=0.001)$, but not in pT2 $(p=0.655)$ and pT4 cancer lesions $(p=0.256)$. For pT3pN0cM0 rectal cancer, the risk of metachronous distant metastases and overall survival
\end{abstract}

H. Ptok · F. Meyer, MD ( $\bowtie) \cdot$ R. S. Croner · B. Garlipp Department of General, Abdominal, Vascular and Transplant Surgery, University Hospital of Magdeburg, Leipziger Str. 44, 39120 Magdeburg, Germany frank.meyer@med.ovgu.de

H. Ptok · F. Meyer, MD · R. S. Croner · I. Gastinger · B. Garlipp Institute of Quality Assurance in Operative Medicine, Otto-von-Guericke University of Magdeburg, Leipziger Str. 44, ZENIT II, 39120 Magdeburg, Germany did not depend on the number of investigated lymph nodes.

Conclusion In rectal cancer, at least $n=12$ lymph nodes are to be minimally investigated. The investigation of fewer lymph nodes is associated with a higher risk of false-negative pN0 findings. In particular, in pT3 rectal cancer, the investigation of more than 12 lymph nodes lowers the risk of false-negative pNo findings. An upstaging effect by the investigation of a possibly maximal number of lymph nodes could not be detected.

Keywords N stage assessment in cancer - M stage . Prospective multicenter observational study • Prognosis · Long-term oncological outcome

\section{Introduction}

For years, retrieval staging has been a paradigm in colorectal cancer surgery. However, recently published analyses have demonstrated that a maximum number of regional lymph nodes is required for accurate tumor but the proportion of node-positive colorectal cancers has not increased over the years, despite steadily increasing numbers of lymph nodes examined in colorectal cancer specimens [1-4]. These analyses generated some doubt regarding the hypothesis that "upstaging" of colorectal cancers occurs as the number of examined lymph nodes increases, and suggest that examination of more than 12 lymph nodes (or as many lymph nodes as possible) might not be beneficial with respect to staging accuracy.

Interestingly, Parsons et al. and Erning et al. found that a higher number of removed lymph nodes is indeed associated with improved long-term survival, even though the proportion of lymph node-positive cancers identified is not increased. This seems to be true for both node-negative and node-positive 
disease. Other authors have reported similar observations $[5,6]$.

Based on a multicenter database of surgically treated rectal cancer patients, the analysis presented here aimed at examining the role of lymph node harvest as a component of adequate histopathological tumor staging as well as the association between the total number of lymph nodes examined and the number of tumor-positive lymph nodes. Moreover, longitudinal changes in lymph node diagnostics during the study period were evaluated.

\section{Methods}

\section{Database}

The analysis is based on data collected within the prospective multicenter observational study on rectal cancer surgery conducted by the Institute for Quality Assurance in Operative Medicine at Otto-von-Guericke University, Magdeburg, Germany (as previously reported [7-16]). Within this database, patient-, tumor-, and treatment-related parameters obtained in patients with newly diagnosed rectal cancer (defined as cancer located within $16 \mathrm{~cm}$ of the anal verge) were recorded. Tumor staging was achieved using CT scan (98\%), EUS (80\%), and MRI (36\%) according to the regional clinical care as established at the time of analysis. In all cases with tumor lesion at the middle and lower third of the rectum, TME was performed (patients with rectal cancer at the upper third underwent PME or TME).

Paraaortic lymphadenectomy (and D3 lymphadenectomy) was not defined as a standard part of oncological resection. However, if this extensive lymphadenectomy was performed, it is was not precisely documented.

According to the guideline-based recommendations, as standard, D2 lymphadenectomy was performed.

Informed consent from all patients is required prior to data entry into the database. Surgical departments of all levels of care throughout Germany are encouraged to enter their data; however, participation in the study is voluntary (the precise number of actively practicing surgeons at the time of data documentation was not registered).

\section{Study population}

For the present analysis, all patients entered into the database from January 1, 2000, to December 31, 2011, were investigated if they fulfilled the following criteria:

i Primary radical surgery for rectal cancer without neoadjuvant treatment;

ii Availability of complete histopathological tumor classification.
Patients who had undergone chemoradiation as indicated were not included in the study. In addition, patients who had undergone non-radical rectal cancer surgery (e.g., local tumor excision) were also not registered.

\section{Assessment of lymph node numbers and lymph node tumor positivity}

Changes in the following parameters over the study period were determined:

- Mean total number of lymph nodes examined per specimen;

- Proportion of specimens containing at least 12 lymph nodes;

- Proportion of specimens with lymph node-positive cancers.

Moreover, the impact of tumor growth through the rectal wall ("pT stage") on lymph node metastases was described. The association between the number of lymph nodes examined per specimen and the rate of detected lymph node-positive cancers was also examined separately for each of the pT stages 2,3 , and 4 .

To account for possible bias inflicted by improvements in lymph node diagnostics implemented during the study period, additional analysis was undertaken using exclusively data from institutions having contributed to the registry throughout the entire period studied (2000-2011). For each institution, the average number of lymph nodes examined per specimen in the years 2000 and 2001 were compared to the corresponding numbers from years 2010 and 2011. Based on these comparisons, the institutions were subdivided into those in which the number of lymph nodes examined had

i increased significantly from $2000 / 2001$ to $2010 / 2011$, and

ii remained unchanged.

Within both groups, the rate of node-positive cancers was compared between the years 2000/2001 and $2010 / 2011$.

Additionally, the association between lymph node metastasis and synchronous distant metastasis as well as metachronous distant metastasis diagnosed after potentially curative rectal resection was studied. For the latter, oncological long-term outcome data for patients recorded between 2005 and 2007 were collected and used to analyze the influence of UICC tumor stage, pN stage, and number of lymph nodes examined (in categories of less than 12 lymph nodes: LN class 1; 12 through 17 lymph nodes: LN class 2 ; 18-23 lymph nodes: LN class 3; or more than 23 lymph nodes: LN class 4) on the emergence of metachronous distant metastases. 


\section{original article}

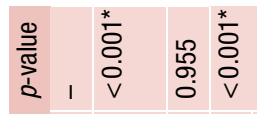

$$
\stackrel{*}{*}
$$

䠉

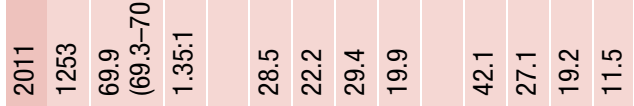

10.

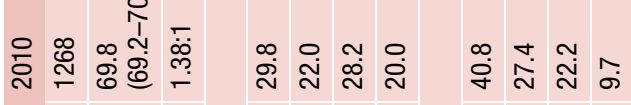

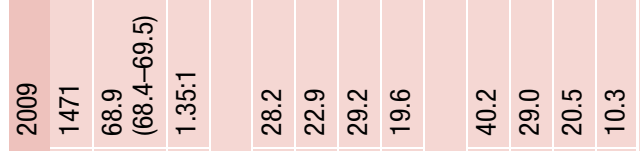

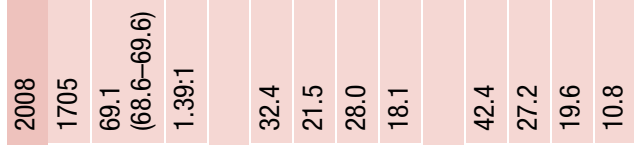

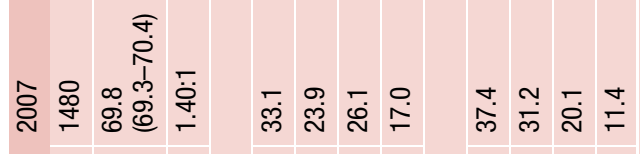

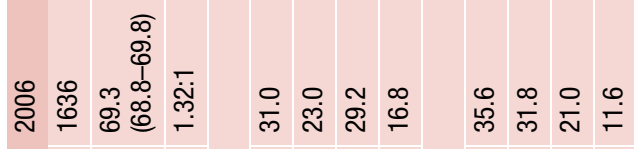

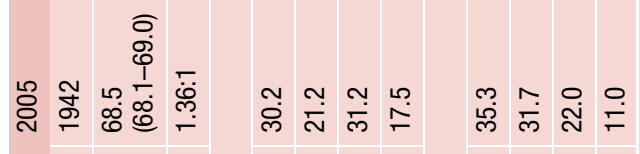

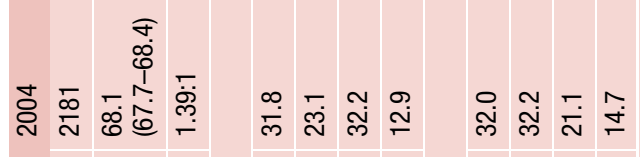

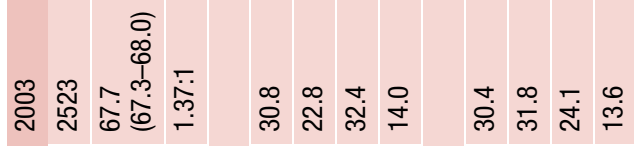

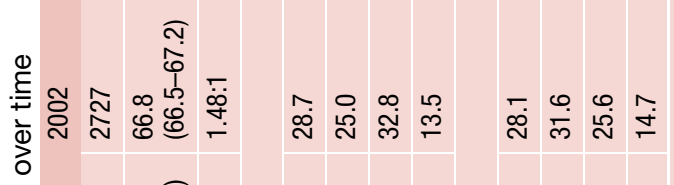

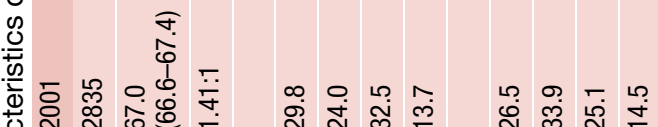

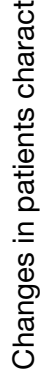

के

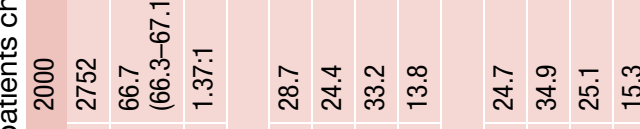

क)

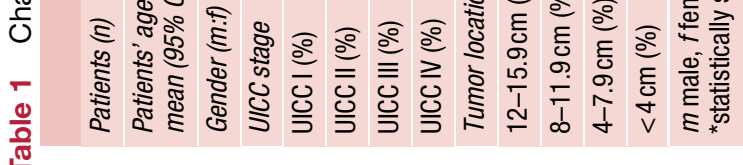

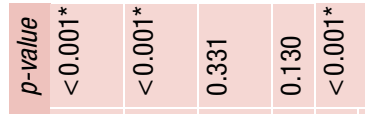

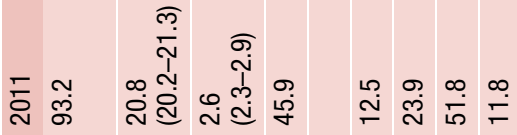

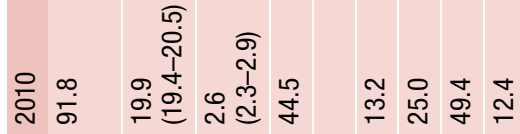

零 官

总

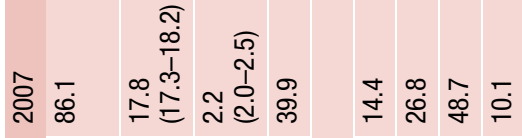

突

票永

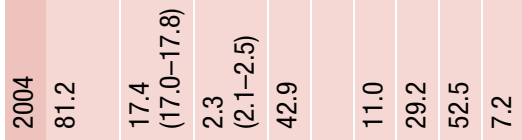

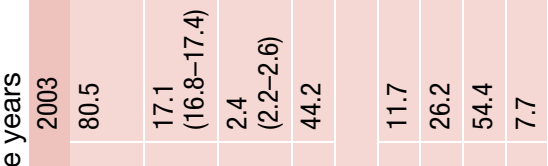

Ф

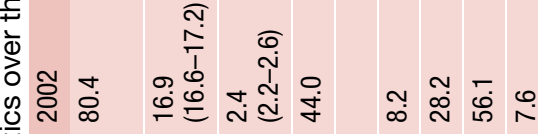

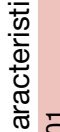

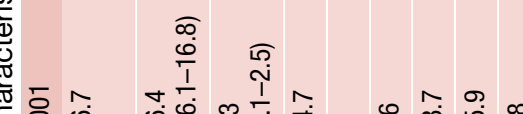

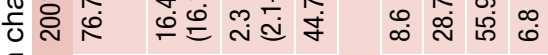

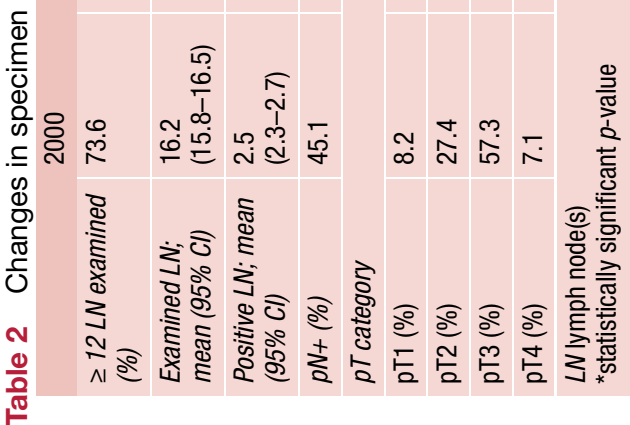


Fig. 1 Frequency of lymph node metastases and clinically apparent distant metastases depending on depth of tumor invasion ( $\mathrm{pT}$ )

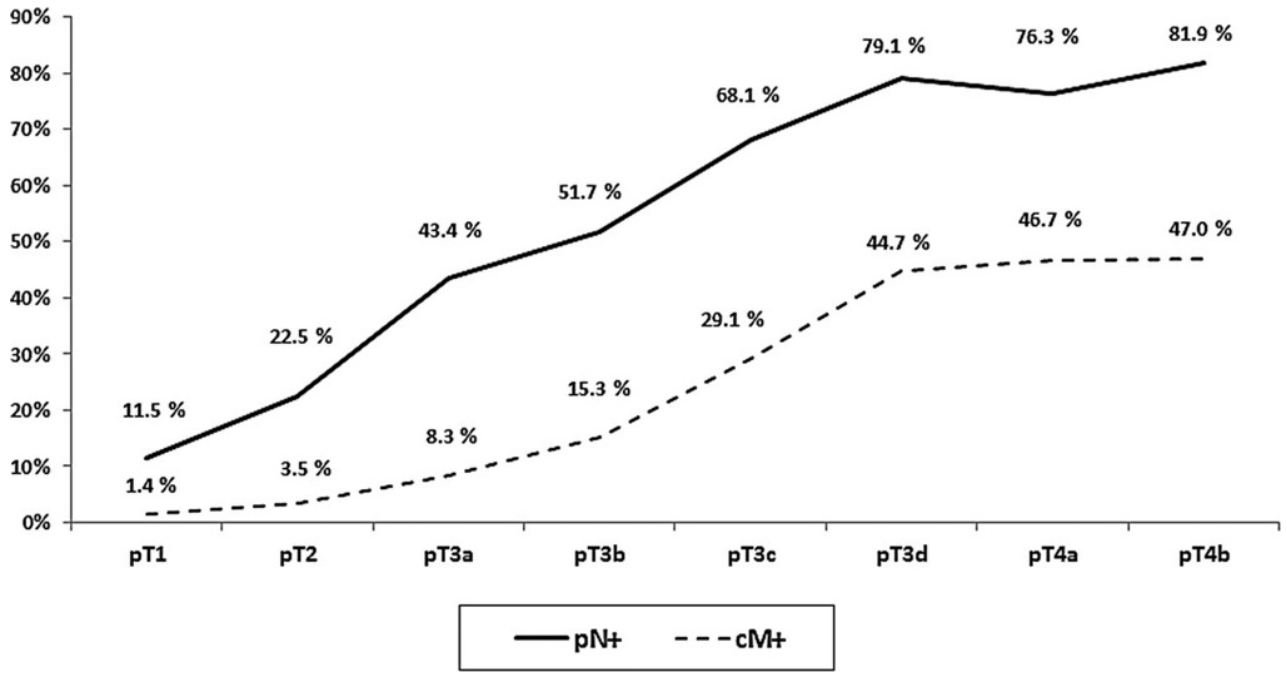

\section{Statistics}

SPSS version 22.0 (IBM ${ }^{\circledR}$ SPSS Statistics, Ehningen, Germany) was used for statistical data analysis. Comparison of continuous parameters was performed using student's $t$-test (in case of two groups) or univariate analysis of variance (one-way ANOVA, in case of more than two groups). Results are presented as mean values and $95 \%$ confidence intervals (CI). $\mathrm{Chi}^{2}$-test was used to compare categorical parameters. A twosided $p$-value of $<0.05$ was considered significantly different.

Univariate analysis of oncological long-term outcomes was performed using the Kaplan-Meier method and results were compared between groups using the log-rank test. Again, a two-sided $p$-value of $<0.05$ was considered significantly different.

\section{Results}

Within the 12-year study period $(01 / 01 / 2000$ through $12 / 31 / 2011$ ), data from $n=23,773$ patients undergoing primary radical resection for rectal cancer without neoadjuvant treatment for whom complete histopathological tumor classification was available were entered into the observational study database. As the proportion of patients who received neoadjuvant treatment and had to be excluded from the analysis increased, a steady decline of patients available for study entry over the 12-year period was observed. Moreover, a significant increase in mean age, proportion of patients in UICC tumor stage IV, and proportion of patients whose tumors were located $>12 \mathrm{~cm}$ from the anal verge was recorded.

In Table 1, patient characteristics over the study period from 2000-2011 are shown.

The rate of rectal cancer specimens from which at least 12 lymph nodes were retrieved and examined increased from $73.6 \%$ to $93.2 \%(p<0.001)$ during the study period. Correspondingly, the mean num- ber of lymph nodes harvested from each specimen increased from 16.2 to 20.8 ( $p<0.001$; Table 2). In spite of these numerical improvements in lymph node diagnostics, only a minor non-significant variation in the rate of patients diagnosed with lymph node-positive cancer was observed $(p=0.130)$. The mean number of tumor-bearing lymph nodes per specimen did not change significantly during the study period regardless of whether all patients or only $\mathrm{pN}+$ patients were analyzed (lymph nodes per specimen 2.2-2.6, $p=0.331$, and 5.2-5.8, $p=0.479$, respectively; Table 2).

Considering the tumor infiltration depth, pT1 and pT4 cancers showed a slight increase (from $8.2 \%$ to $12.5 \%$ and from $7.1 \%$ to $11.8 \%$, respectively) over the study period. Conversely, the rate of pT2 and pT3 cancers decreased (Table 2).

Fig. 1 shows the probability of lymph node metastasis $(\mathrm{pN}+)$ as well as distant metastasis diagnosed on preoperative imaging or intraoperatively as a function of tumor infiltration depth (pT category). Both lymph node metastasis and distant metastasis were significantly more likely to be diagnosed in patients with a more advanced pT stage $(p<0.001)$. The likelihood graphs for lymph node and distant metastases are parallel.

The probability of synchronous distant metastasis as a function of lymph node involvement (pN0 vs. pN1 vs. pN2) can be seen in Fig. 2a for pT categories 1 through 4 . In each of these pT categories, more extensive lymph node involvement was significantly associated with an increase in the probability of distant metastasis diagnosed synchronously with the primary tumor. Moreover, the number of tumor-positive lymph nodes had a significant impact on the probability of distant metastases ( $p<0.001$, Fig. $2 \mathrm{~b}$ ), whereas in node-negative patients, no association between the number of lymph nodes examined and the probability of synchronous distant metastasis was found. The average risk for pN0 patients to be diagnosed with synchronous distant metastases was $4.5 \%$ (Fig. 2c). 
Fig. 2 Frequency of synchronous distant metastasis depending on: a nodal positivity and $\mathrm{pT}$ category of rectal cancer; $\mathbf{b}$ number of positive lymph nodes; c number of examined negative lymph nodes
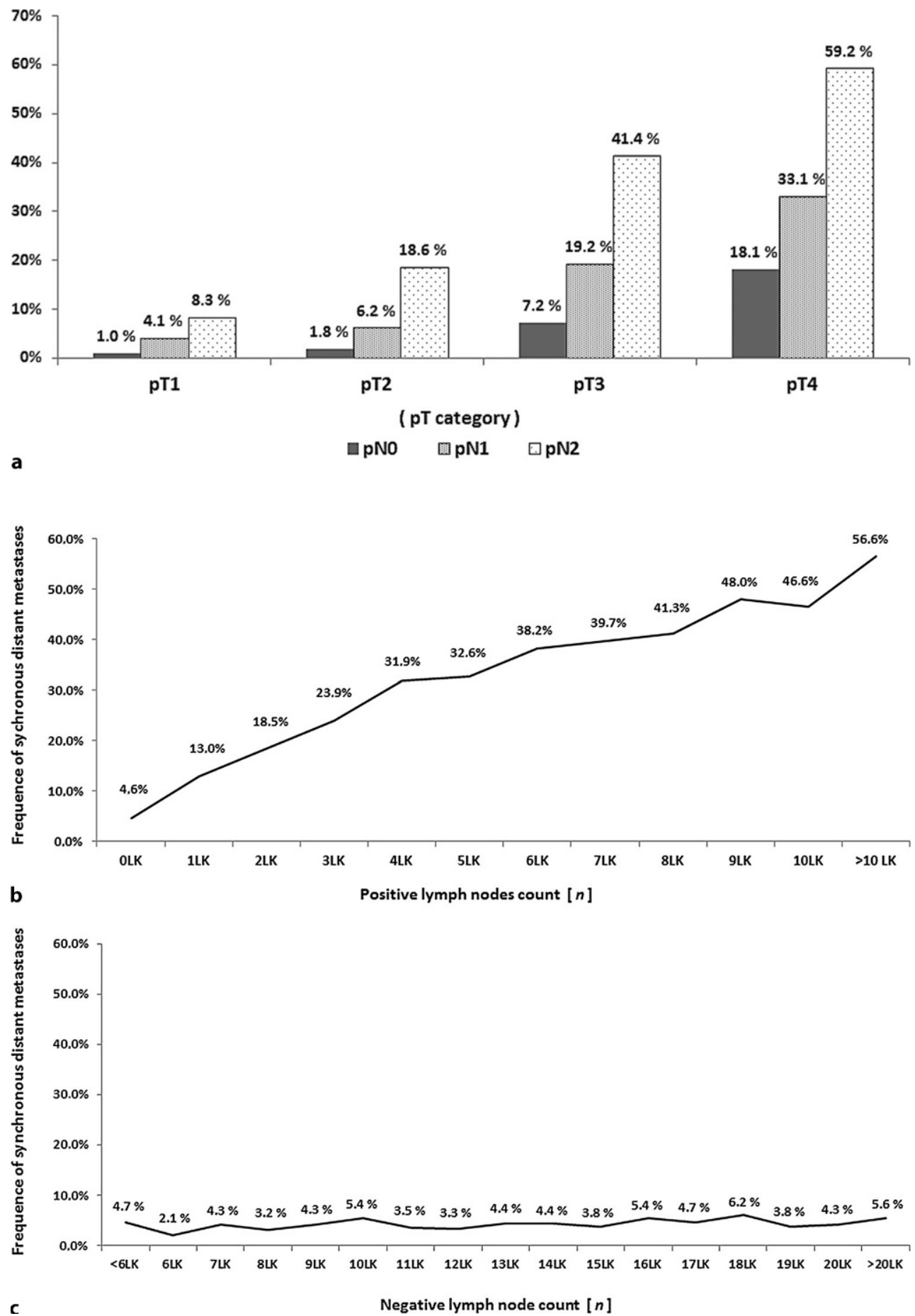

Impact of lymph node involvement on metachronous distant metastasis

Follow-up data of $70 \%$ of the patients entered into the database between 2005 and 2007 ( $n=2733$ of 3930; best follow-up data quality during this time period) were available to analyze the association between initial lymph node involvement and the development of metachronous metastatic disease. Median follow-up was 55.0 (95\% CI: 53.9-56.1) months. Fig. 3a shows the cumulative risk for metachronous metastasis after potentially curative rectal resection as a function of UICC tumor stage, whereas Fig. $3 b$ shows its association with the initially diagnosed $\mathrm{pN}$ category. While patients with lymph node-negative cancer had a $6.7 \%$ cumulative risk of developing distant metastases at 4 years, this was $19.4 \%$ for patients with node-positive cancers $(p<0.001)$. The cumulative risk for patients with pN1 vs. pN2 nodal status was only marginally different ( $18.0 \%$ vs. $21.8 \%$, respectively; $p=0.017$ ). 

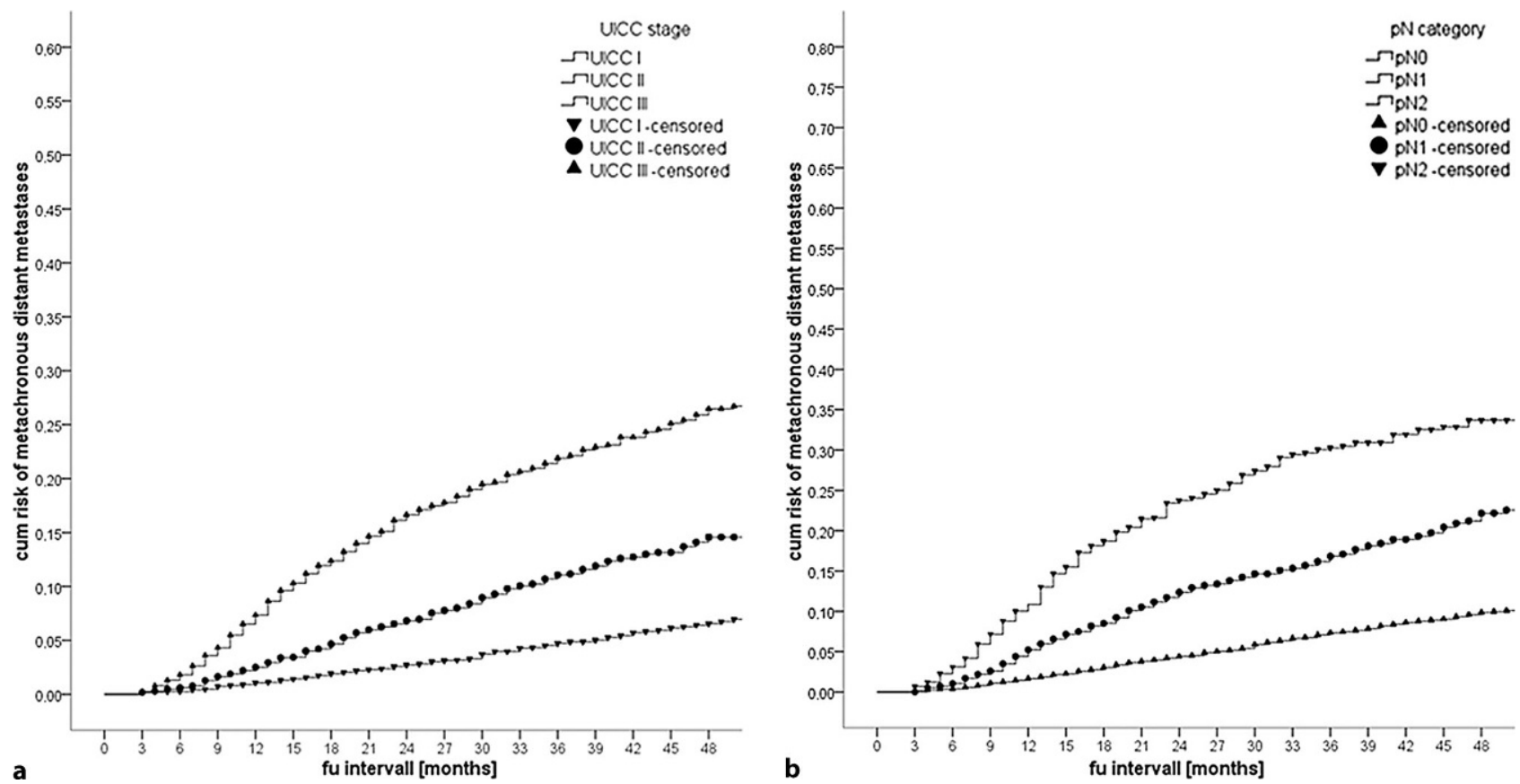

Fig. 3 Cumulative risk for metachronous metastasis as a function of a UICC tumor stage, b pN stage. cum cumulative, fu followup

Impact of the total number of lymph nodes examined on detection of tumor-involved lymph nodes

The association between the total number of lymph nodes examined per specimen and the pN category assigned (pN0 vs. pN1 vs. pN2) is shown in Fig. $4 \mathrm{a}-\mathrm{c}$ for $\mathrm{pT}$ categories 2,3 , and 4 . A significant impact of the total number of lymph nodes on the detection of tumor-involved lymph nodes was found for each of these pT categories (pT2: $p=0.002, \mathrm{pT} 3: p<0.001$, pT4: $p=0.002$ ). $11.6 \%$ of pT2 cancers were diagnosed as being lymph node positive $(\mathrm{pN} 1+\mathrm{pN} 2)$ if less than six lymph nodes were examined whereas this was $25.5 \%$ in patients who had more than 20 lymph nodes examined (Fig. 4a). Among patients with pT3 and pT4 cancers, the rates of lymph node positivity were $33.7 \%$ and $40.5 \%$ if less than six lymph nodes were examined as opposed to $61.1 \%$ and $76.9 \%$ if more than 20 lymph nodes were investigated. Thus, approximately twice as many patients in all three pT categories were diagnosed as having node-positive cancer if they had more than 20 as opposed to less than six lymph nodes examined. Within the groups of patients with pT2 and pT4 cancers, the detection rate of tumor-involved lymph nodes did not increase any further beyond 12 examined lymph nodes $(19.1 \%$ vs. $25.5 \%, p=0.655$; and $76.6 \%$ vs. $77.0 \%, p=0.256$, respectively; Fig. $4 \mathrm{a}, \mathrm{c})$. In contrast, in patients with pT3 cancers, examination of more than 20 nodes did result in a significant increase in detection of positive lymph nodes as compared to examination of only 12 lymph nodes (49.2\% vs. $66.1 \%, p=0.001$; Fig. $4 \mathrm{~b}$ ).
Impact of the total number of lymph nodes examined on metachronous distant metastasis and overall survival

The number of lymph nodes examined in patients with node-negative cancers (pNo) was not significantly associated with the probability of developing metachronous distant metastasis in any of the pT categories pT2, pT3, and pT4. Fig. 5 shows the cumulative risk for distant metastasis in lymph node (LN) classes 1 through 4 (see Patients and methods) for patients initially diagnosed with pT3 pN0 cM0 cancers. The cumulative risk for the development of distant metastasis was $6.2 \%, 8.6 \%, 9.0 \%$, and $15.2 \%$ in LN classes 1 through 4, respectively (log-rank test).

\section{Stage migration}

The impact of the number of examined lymph nodes on the detection of tumor-positive lymph nodes was compared between participating institutions that did (group 1) and did not (group 2) report an increase in the total number of lymph nodes examined per specimen between the years 2000/2001 and 2010/2011. Only pT3 cancers were investigated for this analysis. Results are shown in Table 3. Hospitals of group 1 reported an increase in the mean number of examined lymph nodes from 16.7 to $26.9(p<0.001)$, whereas in hospitals of group 2, the lymph node harvest remained stable (17.6 vs. $18.0, p=0.472)$. Despite the significant increase in the total lymph node number per specimen in group 1 , the mean number of tumorinvolved lymph nodes as well as the rate of node- 
Fig. 4 Association between investigated lymph node count and $\mathrm{pN}$ category in rectal cancer with no neoadjuvant chemoradiation: a pT2 category $(n=6364 ; p<0.001)$; b pT3 category $\quad(n=12,568$; $p<0.001)$; c pT4 category $(n=2027 ; p<0.001)$

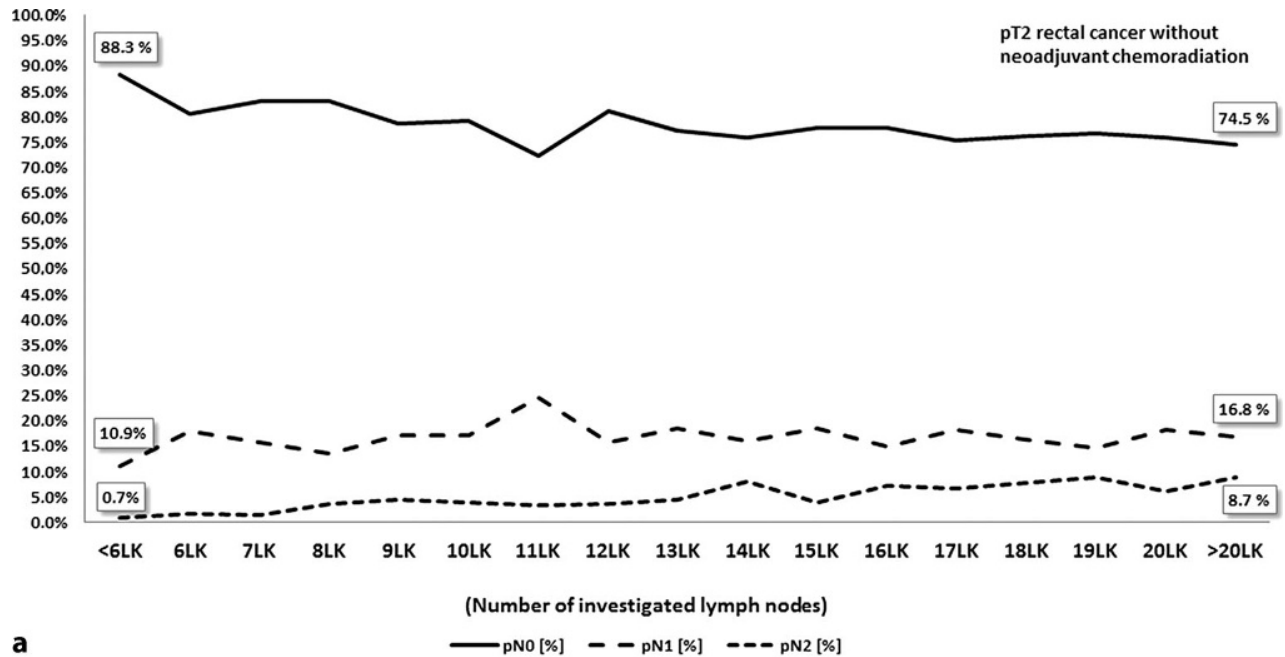

a

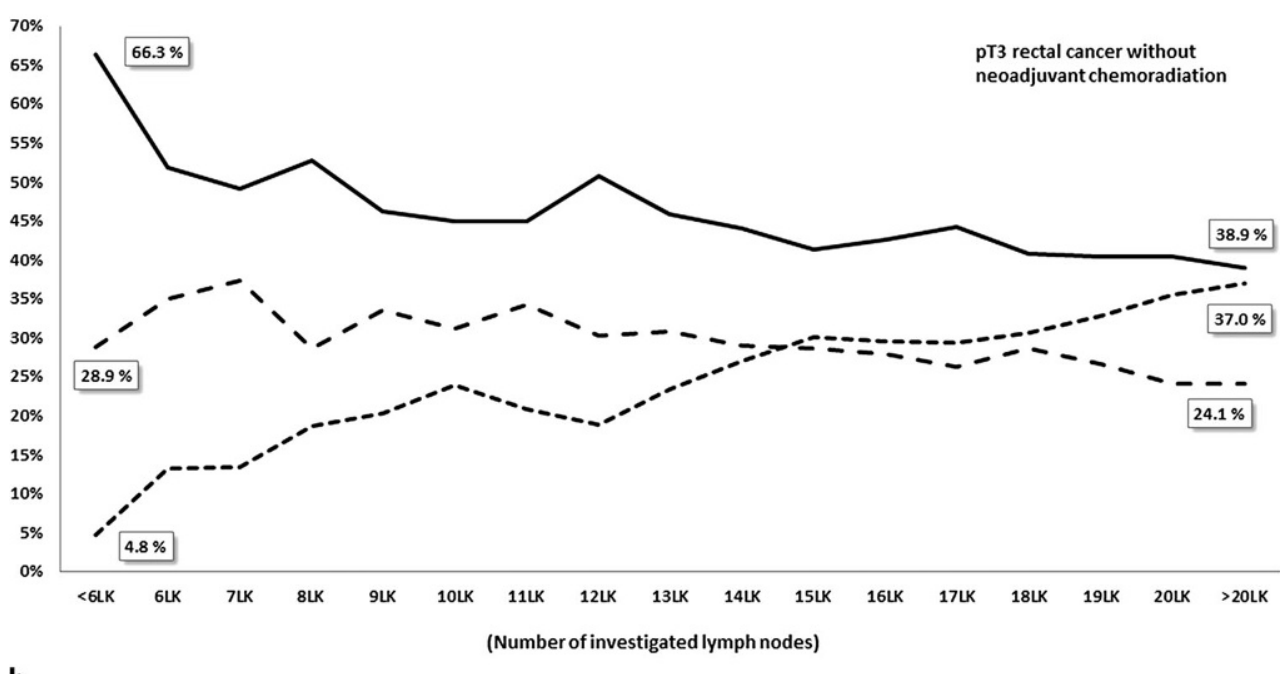

b

- pNO [\%] - - pN1 [\%] - - pN2 [\%]

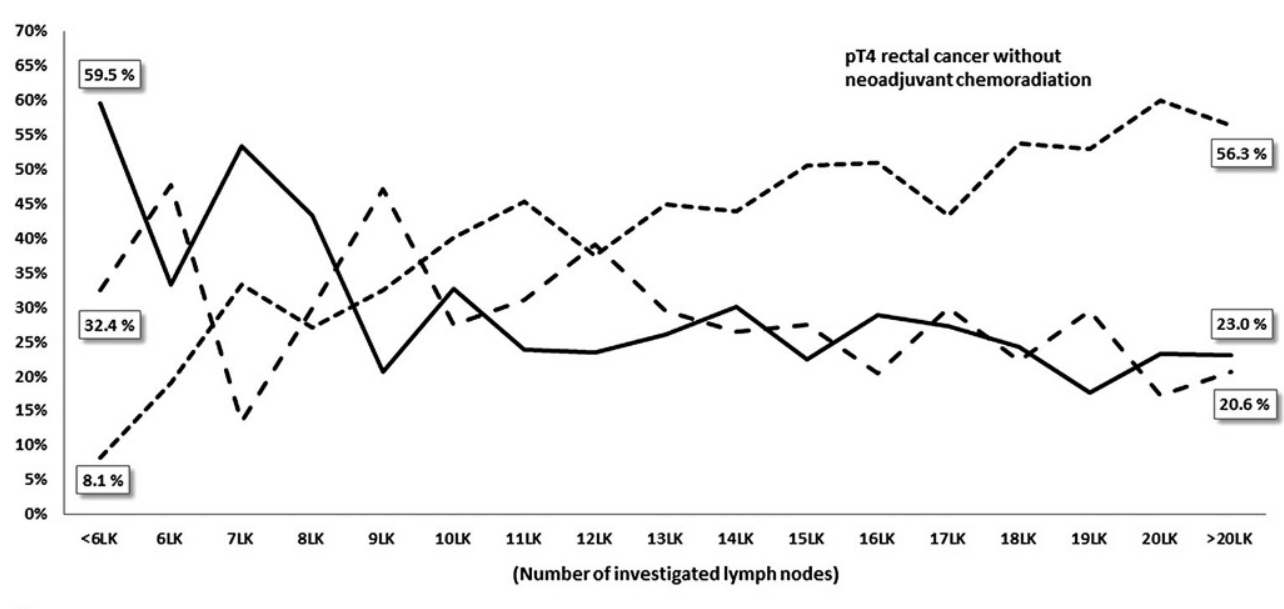

c

- $\mathrm{pNO}[\%] \quad-\quad \mathrm{pN} 1[\%] \quad--\mathrm{pN} 2[\%]$

positive $(\mathrm{pN}+)$ cancers remained unchanged $(57.5 \%$ vs. $55.6 \%, p=0.673$ ). In hospitals of group 2 , there was observed in this group (60.9\% vs. $53.4 \%)$; however, this was not statistically significant $(p=0.097)$. was also no change in the mean number of tumorinvolved lymph nodes ( $p=0.129)$. A tendency towards a decrease in the rate of lymph node-positive cancers 


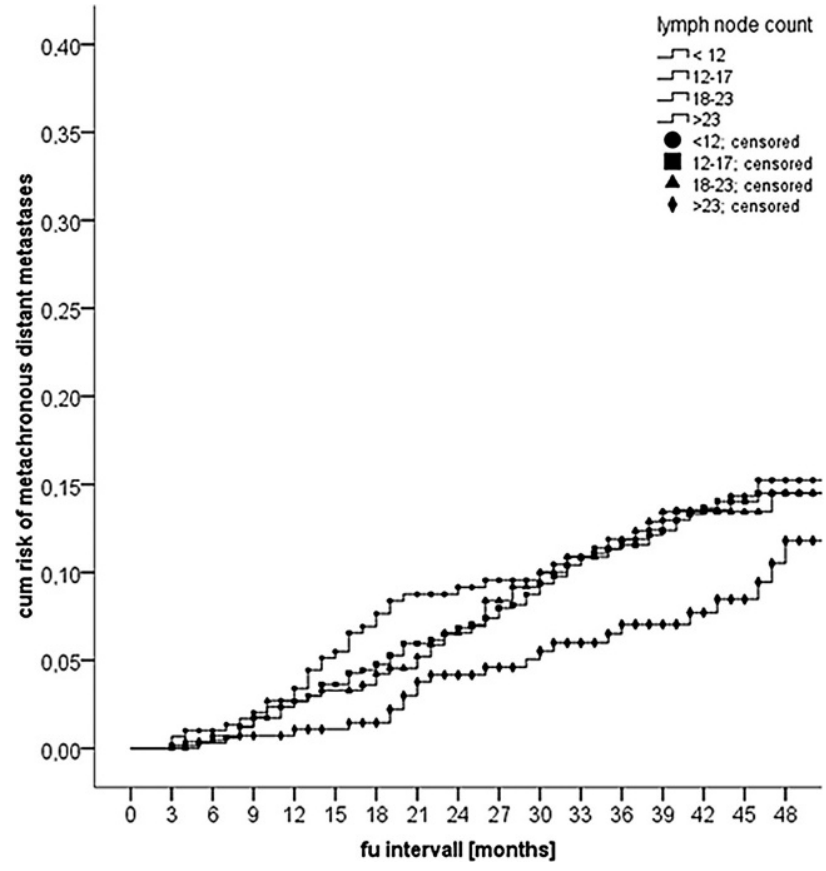

Fig. 5 Risk of metachronous distant metastases depending on lymph node count after curative radical resection of pT3 pNO cM0 rectal cancer. cum cumulative, fu follow-up

\section{Discussion}

Results from analyses encompassing long time periods may be influenced by a variety of factors that may not remain constant during the study period, which, thus, may bias their interpretation. For example, a lack of upstaging in spite of an increased lymph node harvest may also be due to an increase in patients being diagnosed at an earlier disease stage as a result of the growing use of screening colonoscopy in the population analyzed.

The principal results of the analysis of data from an observational study presented here include a significant increase in the number of lymph nodes examined per rectal cancer specimen (Fig. 6) during the study period, which leads to a rising proportion of specimens with a minimum of 12 lymph nodes retrieved. Within pT categories 2 through 4, examination of more lymph nodes leads to identification of more lymph node-positive cancers. Moreover, it has been confirmed that lymph node involvement in rectal cancer is an important indicator for established metastatic disease as well as a predictor for metachronous development of distant metastasis. However, the continuously rising number of lymph nodes examined per specimen during the study period was not associated with a similar increase in the rate of lymph node-positive cancers identified. Thus, it could not be demonstrated that more accurate tumor staging by examination of more lymph nodes leads to consecutive upstaging in rectal cancer. Correspondingly, the cumulative risk of distant metastases following potentially curative resection of
pT3 pN0 cM0 rectal cancer was not dependent on the number of lymph nodes examined.

Similar results of a literature search were presented by Hogan et al. [3], demonstrating relatively little variation in the rate of lymph node-positive rectal cancers, which fluctuated at around $40 \%$ out of a total sample of 240,081 rectal cancers over the past two decades. These authors proposed the concept of a "nodal positivity constant." Similarly, Parsons et al. [1] analyzed 86,394 colon cancer patients included in the SEER database between 1998 and 2008. Despite a significant increase in the number of lymph nodes examined, again, a relatively constant rate of node-positive cancers of approximately $40 \%$ was found. These results are comparable to the $44 \%$ rate of node-positive cancers found in the observational study database presented here and have been confirmed by other authors $[2,4]$, putting the concept of tumor upstaging through more meticulous lymph node examination into question. Interestingly, though, an association between the increase in the number of examined lymph nodes and improved survival, both for lymph node-negative and for lymph node-positive cancers, has been reported [1, 2, 6, 7]; however, this has not been confirmed by all authors [19]. In the current analysis, no association between the number of lymph nodes examined and the rate of metachronous distant metastatic disease was found.

Baxter et al. [20], in an analysis of the SEER data, identified a threshold of 13 examined lymph nodes for identification of lymph node involvement in pT3 colon cancer specimens, exceeding of which does not lead to detection of more lymph node-positive cancers. Correspondingly, the present analysis demonstrates that examination of more than 12 lymph nodes in pT2 and pT4 rectal cancer specimens not treated with neoadjuvant radiotherapy does not increase the detection rate of $\mathrm{pN}+$ cancers. Only in pT3 specimens did we identify a further $11.9 \%$ increase in the detection of lymph node positivity if more than 12 lymph nodes were investigated. Very likely, these observations are the result of the process of lymphatic metastatic spread over time and the distribution of locoregional lymph nodes as well as their pattern of tumor involvement. In a study by Langman et al. [21], $83 \%$ of tumor-involved lymph nodes were located either adjacent to the tumor itself or in the mesorectum proximal to the tumor lesion, whereas only $3 \%$ of involved lymph nodes were found distally to the tumor mass towards the anal verge. Apart from the mesorectum, $16 \%$ of involved lymph nodes were located alongside the superior rectal artery or the inferior mesenteric artery. Only $0.3 \%$ were located elsewhere in the mesentery of the sigmoid colon. The proportion of lymph nodes located in the mesorectum among all identified lymph nodes in the specimen (mesorectum, vascular pedicle, and sigmoid colon mesentery) was only $40 \%$. The median number of lymph nodes identified in the mesorec- 
Fig. 6 Untreated (p)T3 rectal cancer (no neoadjuvant radio[chemo]therapy) of one selected patient with tumor imaging, macroscopic specimen, and histological imaging (HE): a Preoperative MRI. b TME specimen (perspective from ventral: left; from dorsal: right). c T3a stage (according to invasion depth); satellite panel: histopathological image (HE). d Representative imaging for $\mathrm{N}$-stage (lymph nodes increased but not tumor infiltrated); satellite panel: histopathological image (HE)
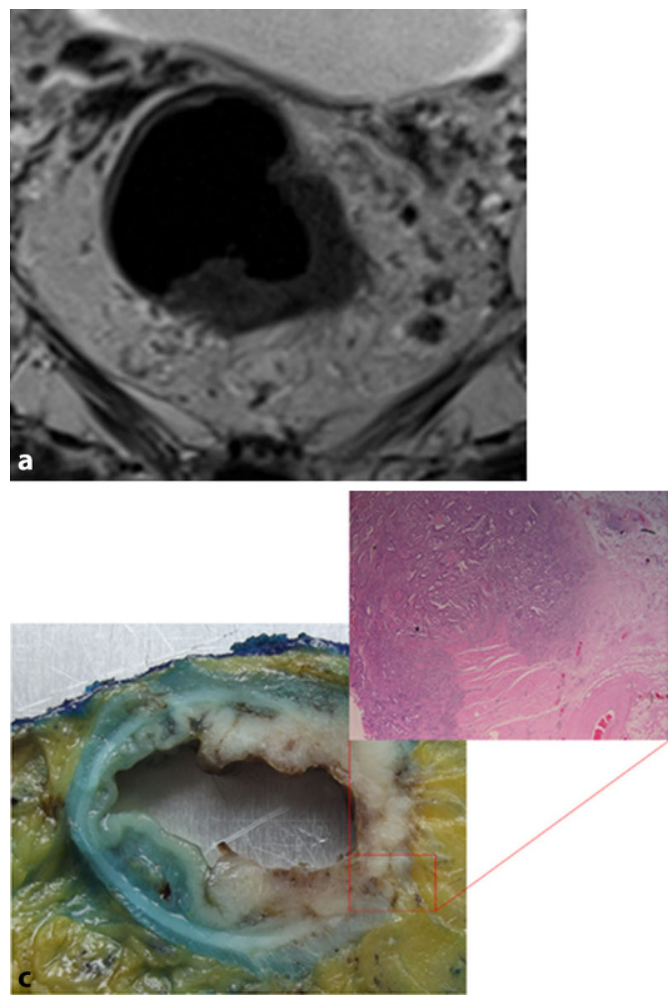
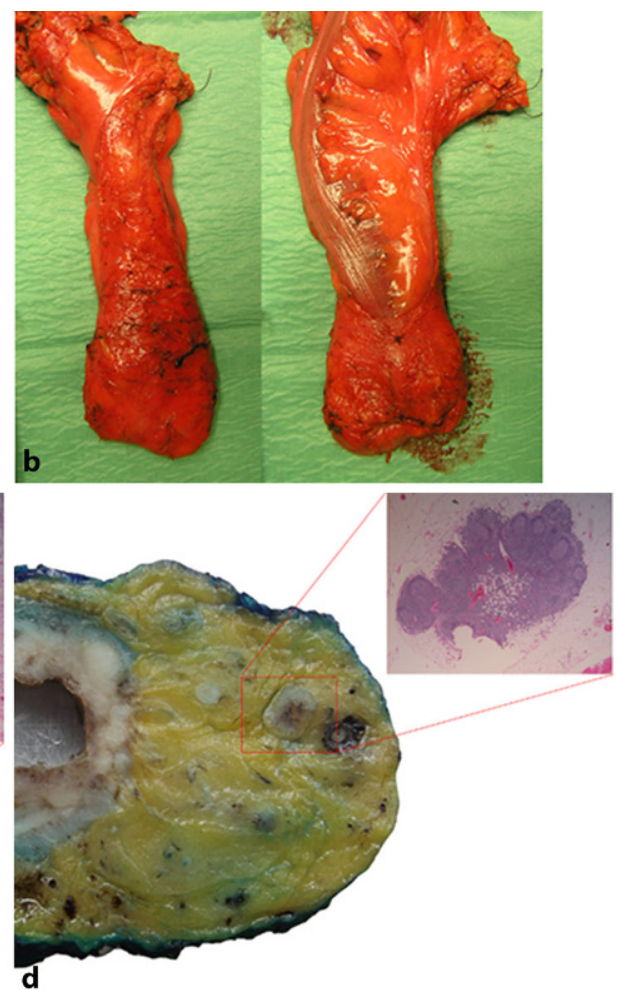

tum was 16. This underscores that examination of 12 mesorectal lymph nodes yields a very high probability of accurate detection or exclusion of lymph node metastases in rectal cancer. In comparison, the chance of detecting tumor-involved lymph nodes outside the mesorectum when all mesorectal lymph nodes are free of tumor appears to be small. This is further highlighted by reports demonstrating that pN0 tumor status in colorectal cancer can be correctly identified in $99.2 \%$ or $98.2 \%$ by the examination of all lymph nodes within $3 \mathrm{~cm}$ or $5 \mathrm{~cm}$ proximally and distally to the tumor, respectively $[22,23]$, even though the rate of false-negative results was higher in rectal cancer compared to colon cancer. Langman et al. [21] also identified lymph node "skip" metastases along the vascular pedicle in $2 \%$ of all $\mathrm{pN}+$ rectal cancer specimens: 9\% of the specimens had tumorinvolved lymph nodes at more than $5 \mathrm{~cm}$ from the tumor even if all lymph nodes located within $5 \mathrm{~cm}$ of the tumor were free of metastases. This translates into a theoretical $11 \%$ false-negative rate if lymph node examination is limited to the peritumoral tissue within $5 \mathrm{~cm}$ of the tumor, mostly in pT3 cancers. Among all specimens analyzed, the false-negative $\mathrm{pN}$ assessment rate would have been $3.3 \%$.

Even though no upstaging effect was found, an increase in the number of lymph nodes examined was associated with improved survival in colon cancer patients in the analysis by Parsons et al. [1] and confirmed by other authors [18, 27]. Some authors [3] have suggested that these counterintuitive observations may be the result of heterogeneity in individual immune responses. A more pronounced immune response may facilitate detection of both non-involved and involved lymph nodes, which may result in more lymph nodes identified by the pathologist in specimens from these individuals. This may lead to bias, as an enhanced immune response may also be associated with better prognosis and improved long-term survival, as demonstrated by Pages et al. [25, 26, 28]. In our analysis, patients with more examined lymph nodes did not demonstrate better long-term survival nor a decreased risk of metachronous metastatic disease, which may be the result of the relatively low proportion of specimens with fewer than 12 lymph nodes found during the study period.

By separately analyzing data from hospitals that did and did not report an increase in the number of examined lymph nodes during the course of the study period, we attempted to determine whether the expected upstaging effect through improved lymph node diagnostics was altered by a tendency towards earlier detection of rectal cancers over the years. Although a more pronounced stage migration towards UICC stage II was found in hospitals in which the number of examined lymph nodes had remained unchanged during the study period $(7.5 \%$ vs. $1.9 \%)$, this difference was not statistically significant. A similar comparative analysis of institutions (A, B, and C) demonstrating different developments in lymph node diagnostics over a defined time period was reported by Storli et al. [4]. In hospitals A and C, an increase in the number of lymph nodes examined per specimen was achieved through optimized surgery (A) or lymph 
Table 3 Prognosis-relevant parameters in lymph node diagnostics comparing surgical departments with increased (group 1) and similar numbers of investigated lymph nodes (group 2) in pT3 rectal cancer during study period

\begin{tabular}{|c|c|c|c|}
\hline & $\begin{array}{l}\text { Time period } \\
2000-2001\end{array}$ & $\begin{array}{l}\text { Time period } \\
2010-2011\end{array}$ & $p$-value \\
\hline \multicolumn{4}{|l|}{ Group 1 (hospitals, $n=20$ ) } \\
\hline Patients $(n)$ & 465 & 247 & - \\
\hline No. of investigated LN; mean ( $95 \% \mathrm{Cl}$ ) & $\begin{array}{l}16.7 \\
(16.1-17.4)\end{array}$ & $\begin{array}{l}26.9 \\
(25.0-28.8)\end{array}$ & $<0.001$ \\
\hline No. of Tu-infiltrated LN; mean (95\% Cl) & $\begin{array}{l}2.9 \\
(2.5-3.4)\end{array}$ & $\begin{array}{l}3.3 \\
(2.5-4.1)\end{array}$ & 0.443 \\
\hline Portion of pN-negative cancer lesions (\%) & 42.5 & 44.4 & \multirow[t]{2}{*}{0.673} \\
\hline Portion of pN-positive cancer lesions (\%) & 57.5 & 55.6 & \\
\hline \multicolumn{4}{|l|}{ Group 2 (hospitals, $n=20$ ) } \\
\hline Patients $(n)$ & 284 & 193 & - \\
\hline No. of investigated LN; mean (95\% Cl) & $\begin{array}{l}17.6 \\
(16.8-18.4)\end{array}$ & $\begin{array}{l}18.0 \\
(17.1-19.0)\end{array}$ & 0.472 \\
\hline No. of Tu-infiltrated LN; mean $(95 \% \mathrm{Cl})$ & $\begin{array}{l}3.3 \\
(2.8-3.9)\end{array}$ & $\begin{array}{l}2.8 \\
(2.2-3.4)\end{array}$ & 0.219 \\
\hline Portion of pN-negative cancer lesions (\%) & 39.1 & 46.6 & \multirow[t]{2}{*}{0.097} \\
\hline Portion of pN-positive cancer lesions (\%) & 60.9 & 53.4 & \\
\hline
\end{tabular}

node preparation $(\mathrm{C})$, whereas in hospital $\mathrm{B}$, the number of examined lymph nodes per specimen as well as the proportion of specimens with at least 12 lymph nodes examined remained unchanged. Still, a decrease in the number of tumor-involved lymph nodes as well as a non-significant trend from UICC stage III towards UICC stage II was found in hospital B. This was also observed in hospital A (optimized surgery) but not in hospital $\mathrm{C}$ (intensified lymph node diagnostics). Our results do not permit conclusion that the expected upstaging effect through more meticulous lymph node diagnostics has been offset by a tendency towards earlier detection of colorectal cancer.

However, as a possible limitation, it needs to be taken into consideration that pathology units use different methods to search for nodes, resulting in difficult comparison of the literature data. In addition, it must also be taken into account that radiotherapy lowers the number of nodes detected and this has good prognostic value. In addition, lymph node harvest is an indirect indicator of appropriate surgery and, thus, of better oncological outcome.

In conclusion, the presented analysis underscores the demand for a minimum number of 12 examined lymph nodes in rectal cancer specimens. Examination of fewer lymph nodes is associated with a higher rate of false-negative $\mathrm{pN}$ staging.

Our data do not demonstrate that maximizing the number of examined lymph nodes per specimen results in tumor upstaging. Despite an increase in investigated lymph nodes, there was no detectable shift of tumor stages.

\section{Conclusion}

- There is a demand for a minimum of 12 examined LN in untreated (no neoadjuvant treatment) rectal cancer specimens.

- Examination of fewer LN is associated with a higher rate of false-negative $\mathrm{pN}$ staging.

- Further increasing the number of examined LN beyond 12 may decrease the false-negative rate, especially in pT3 cancers.

- The data do not demonstrate that maximizing the number of examined LN per specimen results in tumor upstaging.

- Despite an increase in investigated lymph nodes, there was no detectable shift of tumor stages.

Funding Open Access funding enabled and organized by Projekt DEAL.

\section{Declarations}

Conflict of interest H. Ptok, F. Meyer, R.S. Croner, I. Gastinger, and B. Garlipp declare that they have no competing interests.

Ethical standards Research involving human participants and/or animals. The study was performed according to the guidelines and ethical standards of the Declaration of Helsinki for Biomedical Research from 1964 by the World Medical Association and its further amendments, the policy of the local institutional ethics committees, as well as according to the requirements of Good Clinical Research and Good Clinical Practice. Data generation, documentation, and evaluation was performed according to prerequisites of data protection law of the German district Saxony-Anhalt and according to federal law. With regard to the study concept, it can be stated that a (potential) danger for study participants can be definitely excluded. There is no imponderable risk or side effect for the patient. Informed consent. Each patient signed an informed consent form prior to i) surgery, including 
appropriate explanation of the surgical intervention and potential complications, and ii) generation and documentation of data in the patient data registry.

Open Access This article is licensed under a Creative Commons Attribution 4.0 International License, which permits use, sharing, adaptation, distribution and reproduction in any medium or format, as long as you give appropriate credit to the original author(s) and the source, provide a link to the Creative Commons licence, and indicate if changes were made. The images or other third party material in this article are included in the article's Creative Commons licence, unless indicated otherwise in a credit line to the material. If material is not included in the article's Creative Commons licence and your intended use is not permitted by statutory regulation or exceeds the permitted use, you will need to obtain permission directly from the copyright holder. To view a copy of this licence, visit http://creativecommons.org/licenses/by/4.0/.

\section{References and Further Reading}

\section{References}

1. Parsons HM, Tuttle TM, Kuntz KM, Begun JW, McGovern PM, Virnig BA. Association between lymph node evaluation for colon cancer and node positivity over the past 20 years. JAMA. 2011;306:1089-97.

2. van Erning FN, Crolla RM, Rutten HJ, Beerepoot LV, van KriekenJH, Lemmens VE. No change in lymph node positivity rate despite increased lymph node yield and improved survival in colon cancer. Eur J Cancer. 2014;50:3221-9.

3. Hogan NM, Winter DC. A nodal positivity constant: new perspectives in lymph node evaluation and colorectal cancer. World J Surg. 2011;37:878-82.

4. Storli K, Sondenaa K, Furnes B, Leh S, Nesvik I, Bru T, et al. Improved lymph node harvest from resected colon cancer specimens did not cause upstaging from TNM stage II to III. World J Surg. 2011;35:2796-803.

5. Tsai HL, Lu CY, Hsieh JS, Wu DC, Jan CM, Chai CY, et al. The prognostic significance of total lymph node harvest in patients with T2-4N0M0colorectal cancer. J Gastrointest Surg. 2007;11:660-5.

6. Swanson RS, Compton CC, Stewart AK, Bland KI. The prognosis of T3N0 colon cancer is dependend on the number of lymph nodes examined. Ann Surg Oncol. 2003;10:65-71.

7. Katzenstein J, Steinert R, Ptok H, Otto R, Gastinger I, Lippert H, et al. Gender-specific differences of the early postoperative and oncosurgical long-term outcome in rectal cancer-data obtained in a prospective multicenter observational study. Chirurg. 2018;89(6):458-65.

8. Gebauer B, Meyer F, Ptok H, Steinert R, Otto R, Lippert H, et al. How does diabetes mellitus influence early postoperative results and survival in rectal cancer. Dtsch Med Wochenschr. 2018;143(5):e25-e33.

9. Gebauer B, Meyer F, Ptok H, Steinert R, Otto R, Lippert H, et al. Impact of body mass index on early postoperative and long-term outcome after rectal cancer surgery. Visc Med. 2017;33(5):373-82.

10. Garlipp B, Ptok H, Schmidt U, Stübs P, Scheidbach H, Meyer F, et al. Factors influencing the quality of total mesorectal excision. Br J Surg. 2012;99(5):714-20.

11. Ptok H, Marusch F, Meyer F, Schubert D, Gastinger I, Lippert H. Impact of anastomotic leakage on oncological outcome after rectal cancer resection. Br J Surg. 2007;94(12):1548-54.
12. PtokH,MaruschF,MeyerF,WendlingP,WenischHJ, SendtW, et al. Feasibility and accuracy of TRUS in the pre-treatment staging for rectal carcinoma in general practice. Eur J Surg Oncol. 2006;32(4):420-5.

13. Ptok H, Marusch F, Meyer F, Schubert D, Koeckerling F, Gastinger I, et al. Oncological outcome of local vs radical resection of low-risk pT1 rectal cancer. Arch Surg. 2007;142(7):649-55. discussion 656.

14. Ptok H, Meyer F, Steinert R, Vieth M, Ridwelski K, Lippert $\mathrm{H}$, et al. Study Group Colon/Rectum Carcinoma (Primary Tumor Lesion). No prognostic impact of isolated lymphovascular invasion after radical resection of rectal cancer-results of a multicenter observational study. Int J ColorectalDis. 2007;22(7):749-56.

15. Garlipp B, Ptok H, Schmidt U, Meyer F, Gastinger I, Lippert H. Neoadjuvant chemoradiotherapy for rectal carcinoma: effects on anastomotic leak rate and postoperative bladder dysfunction after non-emergency sphincter-preserving anterior rectal resection. Results of the quality assurance in rectal cancer surgery multicenter observational trial. Langenbecks Arch Surg. 2010;395(8):1031-8.

16. Ptok H, Steinert R, Meyer F, Kröll KP, Scheele C, Köckerling F, et al. Long-term oncological results after laparoscopic, converted and primary open procedures for rectal carcinoma. Results of a multicenter observational study. Chirurg. 2006;77(8):709-17.

18. Belt EJ, Te Velde EA, Krijgsman O, Brosens RP, Tijssen M, van Essen $\mathrm{HF}$, et al. High lymph node yield is related to microsatellite instability in colon cancer. Ann Surg Oncol. 2011;19:1222-30.

19. Wong SL, Ji H, Hollenbeck BK, Morris AM, Baser O, Birkmeyer JD. Hospital lymph node examination rates and survival after resection for colon cancer. JAMA. 2007;298:2149-54.

20. Baxter NN, Ricciardi R, Simunovic M, Urbach DR, Virnig BA. An evaluation of the relationship between lymph node number and staging in pT3 colon cancer using populationbased data. Dis Colon Rectum. 2010;53:65-70.

21. Langman G, Patel A, Bowley DM. Size and distribution of lymph nodes in rectal cancer resection specimens. Dis Colon Rectum. 2015;58:406-14.

22. Cserni G, Bori R, Sejben I. Limited lymph-node recovery based on lymph-node localisation is sufficient for accurate staging. J Clin Pathol. 2011;64:13-5.

23. Pusztaszeri $M$, Matter $M$, Kuonen A, Bouzourene $H$. Nodal staging in colorectal cancer: should distant lymph nodes be recovered in surgical specimens? Hum Pathol. 2009;40:552-7.

25. Pagès F, Berger A, Camus M, Sanchez-Cabo F, Costes A, Molidor R, et al. Effector memory T cells, early metastasis, and survival in colorectal cancer. N Engl J Med. 2005;353:2654-66.

26. Pagès F, Kirilovsky A, Mlecnik B, Asslaber M, Tosolini M, Bindea $\mathrm{G}$, et al. In situ cytotoxic and memory T cells predict outcome in patients with early-stage colorectal cancer. JClin Oncol. 2009;27:5944-51.

27. Sarli L, Bader G, Iusco D, Salvemini C, Mauro DD, Mazzeo A, et al. Number of lymph nodes examined and prognosis of TNM stage II colorectal cancer. Eur J Cancer. 2005;41:272-9.

28. Mlecnik B, Tosolini M, Kirilovsky A, Berger A, Bindea G, Meatchi T, et al. Histopathologic-based prognostic factors of colorectal cancers are associated with the state of the local immune reaction. JClin Oncol. 2011;29:610-8. 


\section{Further Reading}

17. Wong SL. Lymph node evaluation in colon cancer assessing the link between quality indicators and quality. JAMA. 2011;306:1139-41.

24. Yao YF, Wang L, Liu YQ, Li JY, Gu J. Lymph node distribution and pattern of metastases in the mesorectum following total mesorectal excision using the modified fat clearing technique. JClin Pathol. 2015;64:1073-7.

Publisher's Note Springer Nature remains neutral with regard to jurisdictional claims in published maps and institutional affiliations. 